Paper-Investigation and Performance Analysis of Some Implemented Features of the ZigBee Protocol and IEEE 802.15.4 Mac Specification

\title{
Investigation and Performance Analysis of Some Implemented Features of the ZigBee Protocol and IEEE 802.15.4 Mac Specification
}

\author{
https://doi.org/10.3991/ijoe.v13i01.5984
}

Shantanu K. Nath, Sayera Aznabi, Nabila T. Islam, Annan Faridi, Wayesh Qarony ${ }^{*}$ American International University (AIUB), Bangladesh

\author{
wayesh@aiub.edu
}

\begin{abstract}
This paper represents wireless sensor networks using numerous topologies of ZigBee. Along with coordinator load, the uses of coordination with such networks were inspected. The investigation has been accomplished via the use of various plots in the OPNET Modeler simulator. The results of the simulation explore the use of coordination with mesh and tree routing to verify the suitability of the topology. It also demonstrates some of the implemented features of the ZigBee protocol and IEEE 802.15.4 MAC specification, using OPNET's ZigBee model suite, like: Mesh routing vs. tree routing, Roaming between Personal Area Network (PANs) \& Failure and Recovery procedures. The simulation has been carried out for 20 minutes to investigate the response of routing topologies on the delay, coordinator load and the MAC Load and the data have been collected. The results indicate that the End to End delay, number of hops and MAC load for mesh routing is lower than the tree routing. The results also show that in case of failure and recovery procedures, the simulated networks perform according to the ZigBee Standard.
\end{abstract}

Key Words - ZigBee, Mesh routing, Tree routing, Roaming between PANs, Failure and Recovery procedures, ACK Mechanism, Beacon parameters, CSMA-CA parameters

\section{Introduction}

In the field of networking, new technologies are emerging on a daily basis. The Wireless Local Area Network (WLAN) and Wireless Personal Area Network (WPAN) are the fastest growing technologies in the field of networking. In the field of short range communication medium the most efficient technology in terms of very low cost implementation, low data rate wireless networks with ultra-low power consumption, ZigBee technology is on the top of the food chain. ZigBee based on IEEE 802.15.4 standard is key enabling technology for the success of both Wireless Sensor Network (WSN) and WPAN [1]. Zigbee Alliance was formed in 2002 as a nonprofit organization. The ZigBee standard has adopted IEEE 802.15.4 as its Physical Layer (PHY) and Medium Access Control (MAC) protocols. The ZigBee Alliance 
Paper-Investigation and Performance Analysis of Some Implemented Features of the ZigBee Protocol and IEEE 802.15.4 Mac Specification

announced availability of Specification 1.0 on June 13, 2005, known as the ZigBee 2004 Specification. [2] The main targeted use for ZigBee is towards applications where low cost, low data rate and greater battery life are the main concern. In many ZigBee operations, the time the wireless device stays active for is very minute. Thus, ZigBee enabled devices are usually very long lasting. [1]. Some recent researches of Zigbee technology has been massively useful in all kinds of sectors such as the Xbee ZigBee, Smart Wireless Lighting[3], Solar power utility, Clean Hands Safe Hands wireless system and in NASA space programs [4].

In this paper, the main objective is to analyze the performance of features of Zigbee like Mesh Routing vs Tree Routing, Roaming between PANs and Failure and Recovery Procedures.

This paper will give the basic idea of how the above features work and how they are important in a network. The analysis is done to further back up the concepts so that the process by which it works can be understood properly.

At first the common features and architecture of Zigbee has been represented, followed by its Network Topologies. Then the applications of Zigbee has been mentioned and finally the results and simulations have been described. After that an overall discussion of the analysis and suggestion for future work have been given.

\section{The Wireless Sensor Network ZigBee}

ZigBee is an uncomplicated packet data protocol which is designed for less weight wireless network. The MAC layer and physical layer of ZigBee are specified with IEEE 802.15.4 standards. The four layers that comprise the architecture of the ZigBee network are Physical layer, MAC layer, Network layer and Application layer. ZigBee protocol holds static, dynamic and mesh topologies. ZigBee protocol provides selfhealing capacity for the network in order to manage defects due to several environmental effects to 'sense and recover from network or connection link faults without the help of human interference. ZigBee has three frequency bands which support different channels. $2.4 \mathrm{GHz}$ band supports 16 channels, 902-928 MHz supports 10 channels and $868-870 \mathrm{MHz}$ supports 1 channel. The maximum limit of data rate for $2.4 \mathrm{GHz}$ is $250 \mathrm{kbps}, 902-928 \mathrm{MHz}$ is $40 \mathrm{kbps}$ and $868-870 \mathrm{MHz}$ is $20 \mathrm{kbps}$ [5].

\subsection{The Features of ZigBee [6]}

- Less Data Transfer Rate

- More cost effective

- Small time delay

- Low power consumption

- Adjustable effective band

- Dependable

- Networks Quantity

- More Secured 
Paper-Investigation and Performance Analysis of Some Implemented Features of the ZigBee Protocol and IEEE 802.15.4 Mac Specification

\subsection{Architecture of ZigBee[7]}

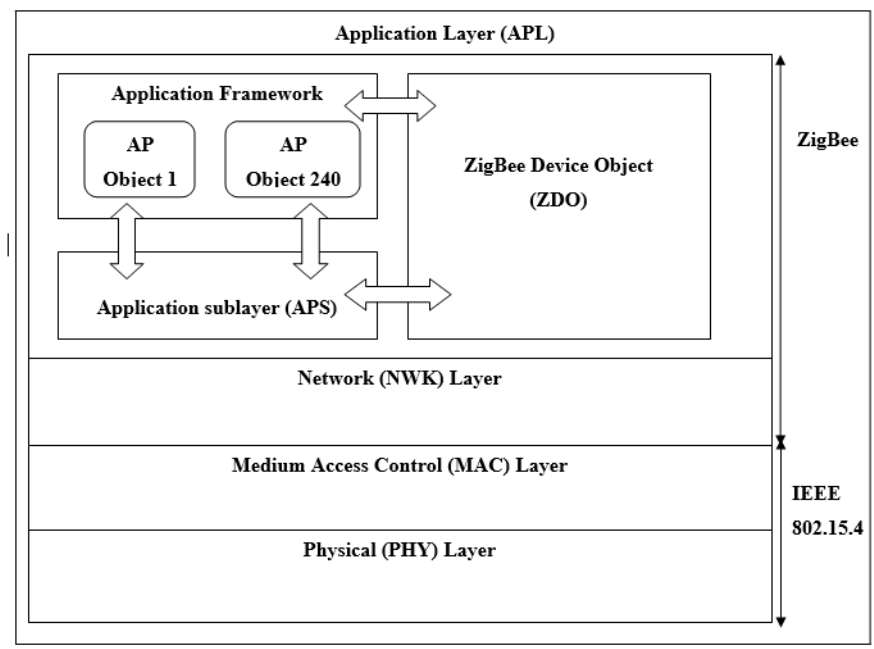

Fig. 1. Architecture of ZigBee

Application layer is the highest layer and it carries the application objects [8]. Every application on ZigBee runs on this layer. There is a special application on every ZigBee device which is called ZigBee Device Object (ZDO). ZigBee specification divides the application layer into three sub layers.

1. The Application Objects commands and handles the protocol layers in ZigBee device. It is a software piece that controls the hardware. Single application object appoints unique end point number that other APO's can use an addition to the network device address to communicate with it [9].

2. ZigBee Device Object plays three operations: service discovery, security and binding [10].

3. Application Support Sub layer (APS) has procedures for outgoing/incoming frames in consideration of sending/receiving the frames safely and constructing/managing the cryptographic keys. Moreover, ASP performs the following functions [8].
(a) Organize key
(b) Carrier key
(c) Advance device
(d) Discharge key
(e) Request key
(f) Switch key
(g) Entity Authentication
(h) Permission configuration table

Network layer provide interface between application layer and MAC layer. This layer is implemented by ZigBee alliance which is an organization of companies which 
Paper-Investigation and Performance Analysis of Some Implemented Features of the ZigBee Protocol and IEEE 802.15.4 Mac Specification

work together for the better ZigBee standards. Network layer gives routing, network management, security management and so on. Self-healing capacity for which ZigBee is popular is provided from this layer [11].

Medium Access Control Sub-Layer is responsible for addressing of data to decide either where from the frame is coming from or going. Besides it gives multiple access control for example CSMA/CA which allows reliable data sending [12].

The Physical Layer is the nearest layer to the hardware. It plays the role of controlling and communicating via radio transceiver directly. It is responsible for all the works which enter to the ZigBee hardware. This layer provides channel selection for avoiding radio interference and also allows data exchange with MAC layer [13].

\subsection{ZigBee Network Topology[14]}

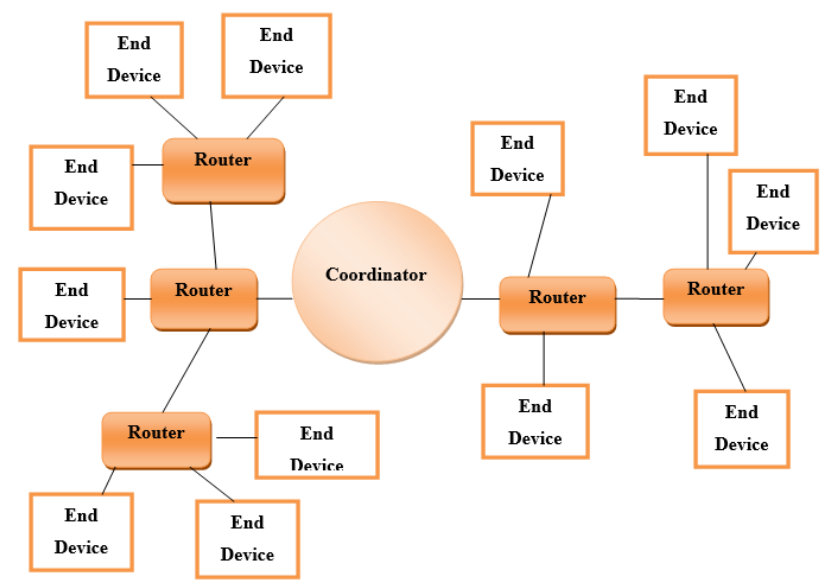

Fig. 2. ZigBee Network Topology

The ZigBee sensor network can support three network topologies [15]. They are

- Star Topology

- Tree Topology

- Mesh Topology

Star Topology: Group of devices is connected to the single coordinator in star topology. It supports up to 6000 devices [11]. This kind of topology is simple and all the packets must go through the coordinator. The coordinator is in charge for the operations of the network. When coordinator fails due to some technical liability, the entire network stops to work as the coordinator handles the traffic. So the coordinator is the bottleneck of this topology. Moreover, this decreases the network efficiency when number of nodes increases. So this type of topology does not give reliable transmission.

Tree Topology: In tree topology, the coordinator is considered as the root of the tree. The router or the end devices can be coupled to the coordinator and these can be extended like a tree [16]. In this topology the routers or the coordinators are parents 
Paper-Investigation and Performance Analysis of Some Implemented Features of the ZigBee Protocol and IEEE 802.15.4 Mac Specification

and end devices are the children. Here the end device does not have the capability to relay messages, so they can never function like parents. When a node needs to transmit a message to another node, the message is transmitted to the parent node and the parent node ensures that the message reaches to that specific node. This topology is quite reliable. If the parent nodes are disabled, the children nodes within the parent nodes cannot communicate with others, even if there are two close nodes topographically.

Mesh Topology: Mesh topology is the most flexible and widely used among other topologies. In this topology, packets go through multiple numbers of hops to reach their destination. Adding more devices in the network increases the coverage area. As a result, dead zones can be removed. Attachment and elimination of a device is easy. When a router fails to act, ZigBee's self-healing mechanism finds other ways to relay the message. It is the most trustworthy topology [11].

\section{Comparison of ZigBee with other Technologies}

ZigBee has some competing Technologies. They are: Z-wave, Insteon, Waveins, Bluetooth and Wi-Fi.

Table 1. Comparisons of ZigBee with similar technologies [17]

\begin{tabular}{|c|c|c|c|c|c|c|}
\hline Features & ZigBee & Z-wave & Bluetooth & Waveins & WiFi & Insteon \\
\hline Frequency & $\begin{array}{l}868 \mathrm{MHz} \\
915 \mathrm{MHZ} \\
2.4 \mathrm{GHZ}\end{array}$ & $\begin{array}{l}868 \mathrm{MHz} \\
908 \mathrm{MHz} \\
2.4 \mathrm{GHz}\end{array}$ & $2.4 \mathrm{GHz}$ & $\begin{array}{l}433 \mathrm{MHz} \\
868 \mathrm{MHz} \\
915 \mathrm{MHz}\end{array}$ & $\begin{array}{c}2.4 \mathrm{GHz} \\
5 \mathrm{GHz}\end{array}$ & $904 \mathrm{MHz}$ \\
\hline Modulation & $\begin{array}{c}\text { BPSK } \\
\text { O-QPSK }\end{array}$ & FSK/GFSK & FHSS & GFSK/PSK & $\begin{array}{c}\text { QPSK } \\
\text { COFDM } \\
\text { QAM }\end{array}$ & FSK \\
\hline $\begin{array}{c}\text { Error } \\
\text { Control }\end{array}$ & $\begin{array}{c}\text { CRC (16 } \\
\text {-bit) }\end{array}$ & CRC (8-bit) & CRC (16-bit) & $\mathrm{BCH}$ & $\begin{array}{c}\text { CRC (32- } \\
\text { bit) }\end{array}$ & $\begin{array}{c}\text { CHECKS } \\
\text { UM }\end{array}$ \\
\hline Range & $10 \mathrm{~m}-100 \mathrm{~m}$ & $30 \mathrm{~m}-100 \mathrm{~m}$ & $10 \mathrm{~m}$ & $\begin{array}{l}200 \mathrm{~m}- \\
1000 \mathrm{~m}\end{array}$ & $100 \mathrm{~m}$ & $45 \mathrm{~m}$ \\
\hline $\begin{array}{c}\text { Network } \\
\text { Size }\end{array}$ & 64000 & 232 & 8 & unknown & 2007 & 256 \\
\hline $\begin{array}{c}\text { Power } \\
\text { Consumption }\end{array}$ & Very Low & Low power & Medium & Ultra-low & High & $\mathrm{NA}$ \\
\hline
\end{tabular}

\subsection{OPNET}

OPNET Technologies Co., Ltd., was established in 1991 which specializes in designing, manufacturing and marketing the carrier-class telecommunication network access and optical transport equipment. OPNET provides services and solutions for worldwide customers ranging from incumbent fixed-network operators, mobile operators, cable TV operators, utilities companies, enterprises to military entities. Their international customers are in EMEA, North/South America and APAC regions. In addition to providing communications network equipment, they also support network planning, installations, trainings and maintenance services for their customers. It is an ISO-9001 certified company [18]. 
Paper-Investigation and Performance Analysis of Some Implemented Features of the ZigBee Protocol and IEEE 802.15.4 Mac Specification

The OPNET ZigBee conducts different process models:

- ZigBee MAC Model

- ZigBee Application Model

- ZigBee Carrier Sense Multiple Access/Collision Avoidance Model

- ZigBee network Model [19]

Full Function Device (FFD) is a node that can be used for transmitting and receiving data, but it can also route data from other nodes. This device can act as a coordinator (master).

Reduced Function Device (RFD) is a device that has a reduced level of functionality. Usually it is an end node which may be a sensor or switch. RFDs can only communicate with FFDs as they contain no routing functionality.

\subsection{Applications of ZigBee Wireless Sensor Networks}

- Medical Care

- Fire Emergency

- Smart Energy

\section{Communication Procedure and Routing Protocols}

In ZigBee, data can be sent in the following ways:

- From a device to the coordinator

- From a coordinator to a device

- Between any two devices

Sending from a device to the coordinator and sending from the coordinator to the device are possible in a star topology. Seeding between any two devices is not possible. In peer to peer network all these three types are possible. Besides transmission can be possible two ways depending on whether beacon transmission is granted or not [20]. Each network has 16bit Pan ID and all devices in ZigBee are assigned to the PAN ID

Basically in ZigBee network, there are two types of routing algorithms.

- AODV (Ad hoc On Demand Distance Vector)

- Tree based routing algorithm

An advanced routing algorithm is declared by investigate the network configure, topology, formation, address assignment and routing protocol of ZigBee [21].

From a Device to the Coordinator: At first the device hears from beacon. While searching for the beacon, it synchronizes to the super frame structure. Through this step it is possible to find out the start time and end time contention access period. The next device will connect with its peer for sharing the channel. When it turns on, it will send the data to the coordinator. If it is not optional, the coordinator may give feedback with an acknowledgement [15]. 
Paper-Investigation and Performance Analysis of Some Implemented Features of the ZigBee Protocol and IEEE 802.15.4 Mac Specification

From a Coordinator to a Device: Coordinator contains the data that has to be transmitted to the device and it is shown in the pending address fields of its beacon. Devices aim at the beacons and decode the pending address fields [15]. If a device finds its address list among the pending address fields, it becomes aware of receiving data from the coordinator. However, a Data Request Command is issued to the coordinator. The coordinator answers it with an acknowledgement. If data is sent to the device, the data will be transmitted. When the confirmation is not optional, the device will respond with an acknowledgement.

Between any two devices: It cannot be preordained in which direct communication will be held between two devices of the network [15]. However, in this situation suitable methods are applicable for transmission. One method is mutual synchronization technique and the other one is direct transmission using un-slotted CSMA-CA [22]. But both techniques have drawbacks. The synchronization technique is very hard to implement. Correspondingly, in direct communication the throughput performance of the PAN may be weakened.

\section{Node Descriptions}

ZigBee Coordinator: The heart of a network which is the coordinator is also the node which initializes the network, selects the proper channel and gives permission to other devices to connect with its network [22]. It can also responsible for routing traffic and suggest which routing technique should be used to transfer data into different nodes. The co-coordinator provides message routing (in tree topology). There may be one PAN coordinator in the network which has the ability to communicate with any device in the network. In every topology there is a coordinator.

ZigBee Router: Router is a fully functional device that supports data routing functionality and works as an intermediate device to add with different components of the network and forwards messages between remote devices and multi hop paths [22]. A router can establish communication with other routers and end device. It is also connected to the main power supply. Routers are used in both tree and mesh topologies. In tree topology routers are located at such positions of the network that grants access to messages to be transfer up and down the tree. In a Mesh topology, the router can be located anywhere in which a message passing node is required.

ZigBee End Device: The power saving features of ZigBee can be mainly credited to the end devices. It is also known as a Reduced Functional Device (RFD) [22]. It is possible because when they are not active, they can go to sleep mode. As a result, this increases the battery life of the end device. The primary tasks of an end device at the network level are to transmit and receive messages. It cannot reply to messages and does not allow other nodes to connect to the network through them. Also it can communicate with the network through the router or directly with the coordinator.

Network setting: The major task of network layer gives the proper ability of using MAC sub-layer and contributes exact port for using through the next upper layer.

In Beacon enable network, coordinator plays an important role. It analyzes a portion of time in which transmission can be taken place and interval of time where every node associated to it should go to sleep. In this network time are divided into 
Paper-Investigation and Performance Analysis of Some Implemented Features of the ZigBee Protocol and IEEE 802.15.4 Mac Specification

super frames. A super frame is a time gap that has active time period and inactive time period [23].

The Physical Layer is the closest layer to the hardware where controlling and communication are obtained through it using radio transceiver. All the work that pass to the ZigBee hardware is done by this layer. The characteristic of the physical layer are activation and deactivation of the radio transceiver, link quality detection (LQI), channel selection, clear assessment (CCA), energy detection (ED) and both sending and accepting packets over the physical medium [24].

\section{MAC parameter}

The layer that is taken from the IEEE 802.15.4 provide services to the network layer and also that is a part of the ZigBee stack level. With the help of addressing data where the frame is advancing or returning is represented with the Mac layer. The layer provides multiple access point (for example CSMA-CA)

\subsection{CSMA-CA mechanism}

CSMA-CA is used by the network node to identify the channel and it checks whether networks are idle or not before sending data [30]. In this topic the back off has becomes vital. Collisions and packet loss are the serious problems here. Back off determines the estimation of time due to waiting before transmission during the channel is busy or after collision. A node which spends time for back off unnecessarily causes hampering throughput and causing delay and greater power absorption. On the other hand, the node that back off early can do many Clear channel assessment (CCAs).it is important to choose the right method of back off for improving network performance. The important factor of back off which effects the network efficiency is the area of back off exponential (BE) value and how many times CCA performs before transmission, the strategy for choosing and repairing BE value during all successful transmission and how many times the node takes attempt to send a packet. In PAN coordinator all the devices have back off periods adjusting with it. As a result, every time the device wants to send data it locates the boundary of the next back off period and then spends time for random numbers of back off. In case that the channel is busy, with the help of this random back off, the device will wait for another random number of back off periods before attempting to enter into the channel. Whenever it finds the channel isd not busy, the device will find next available back off boundary and will send the packet. Acknowledgement and beacon frames are not applicable in CSMACA mechanism [31]. 
Paper-Investigation and Performance Analysis of Some Implemented Features of the ZigBee Protocol and IEEE 802.15.4 Mac Specification

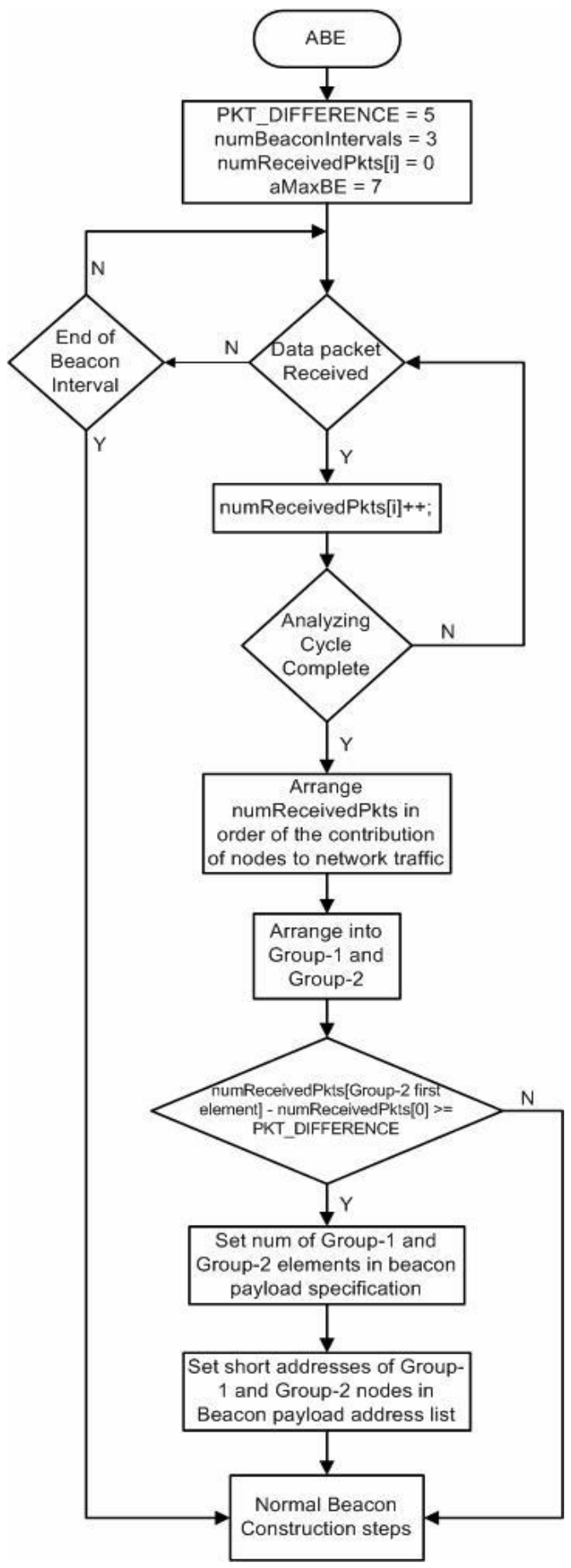

Fig. 3. CSMA-CA Mechanism [32] 
Paper-Investigation and Performance Analysis of Some Implemented Features of the ZigBee Protocol and IEEE 802.15.4 Mac Specification

\subsection{ACK Mechanism}

ACK is a signal passed between communicating processes or computers to signify acknowledgement, or receipt of response, as part of a communications protocol. Medium access control (MAC) is proposed for short-range high-speed wireless personal area networks (WPANs) in the IEEE 802.15.3a task group. In the MAC, three acknowledgment (ACK) mechanisms are adopted during channel time allocation for error control over the error-prone wireless channel. [33]

\subsection{Result and Simulation:}

The objective of this thesis is to demonstrate some of the implemented features of the ZigBee protocol and IEEE 802.15.4 MAC specification, using OPNET's ZigBee model suite, like:

- Mesh Routing vs Tree Routing

- Roaming between PANs

- Failure and Recovery Procedures

\section{Mesh Routing vs Tree Routing}

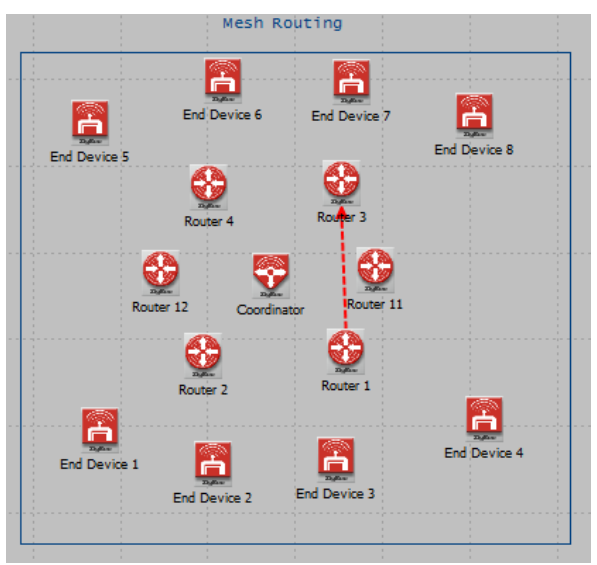

Fig. 4. Mesh Routing [26]

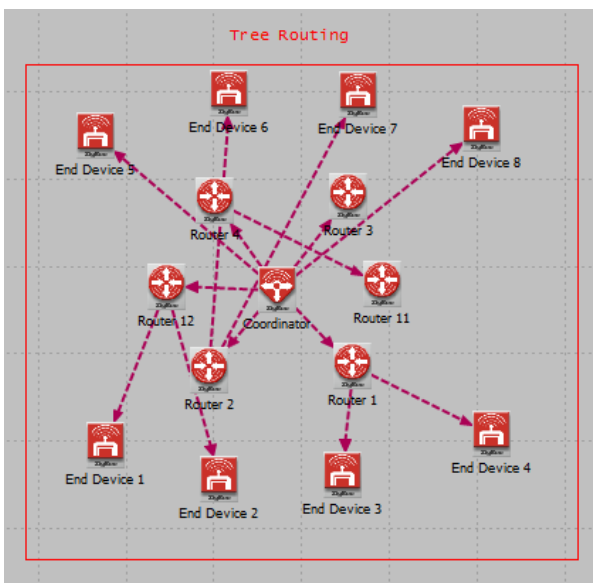

Fig. 5. Tree Routing [26] 
Paper-Investigation and Performance Analysis of Some Implemented Features of the ZigBee Protocol and IEEE 802.15.4 Mac Specification

\section{Result:}

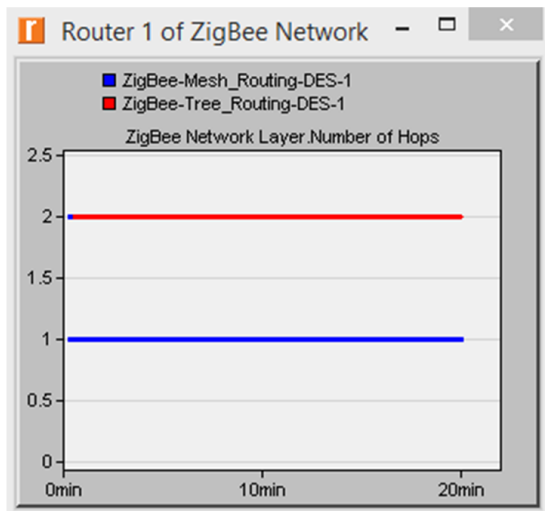

a) Showing Router 1 for ZigBee network

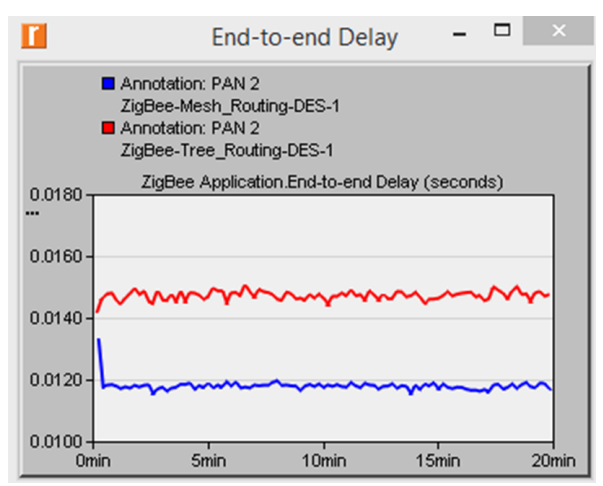

b) End to End delay

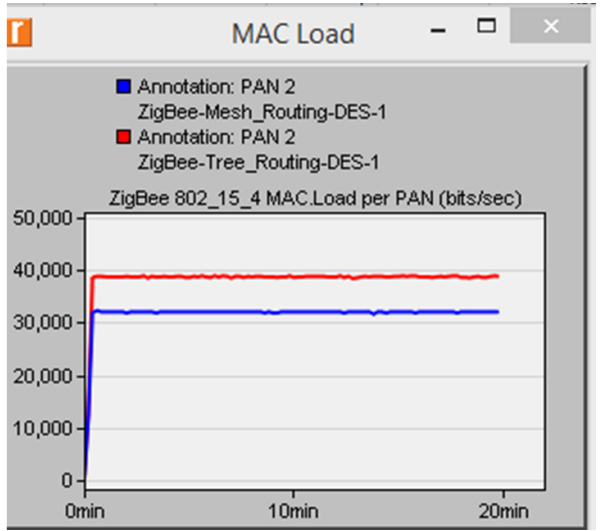

c) Mac load

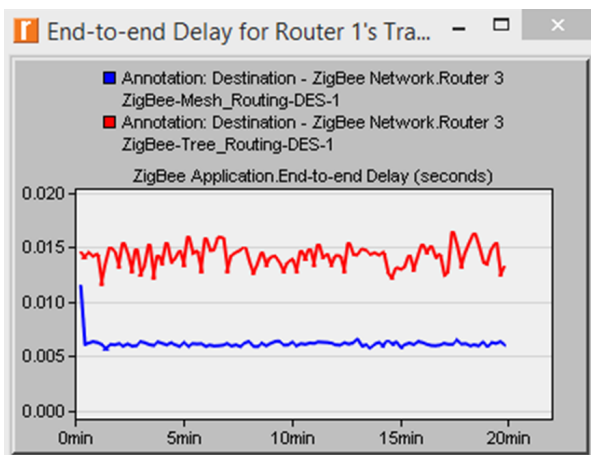

d) End to end Delay Router 1

Fig. 6.

Table 2. Some Features of Mesh \& Tree Topology

\begin{tabular}{|c|c|c|}
\hline Topology & Mesh & Tree \\
\hline Scalability & Yes & Yes \\
\hline Synchronization & No & Yes \\
\hline Inactive period & ZEDs & All nodes \\
\hline $\begin{array}{c}\text { Guaranteed } \\
\text { Bandwidth }\end{array}$ & No & Yes(GTS) \\
\hline Redundant paths & Yes & No \\
\hline $\begin{array}{c}\text { Routing Protocol } \\
\text { Overhead }\end{array}$ & Yes & No \\
\hline $\begin{array}{c}\text { Commercial } \\
\text { Available }\end{array}$ & Yes & No \\
\hline \multicolumn{2}{|c}{}
\end{tabular}


Paper-Investigation and Performance Analysis of Some Implemented Features of the ZigBee Protocol and IEEE 802.15.4 Mac Specification

Number of hops: The graph (Fig. 6a) indicates the number of hops network traffic, originating in Router 1; it takes to reach its destination. The red line represents Tree_Routing while the blue is for Mesh_Routing. Note that both lines begin at two hops, but the Mesh_Routing line quickly changes to one hop for the remainder of the simulation.

Global End-to-End(ETE) Delay: In the graph (Fig. 6b) the red line indicates ETE Delay for Tree_Routing while the blue line indicates ETE Delay for Mesh_Routing. The ETE Delay for Mesh_Routing is lower due to the ability of the mesh routing process to find more efficient routes than tree routing for some of the traffic. For some nodes, the tree based route will be the most efficient route, resulting in only a minor overall improvement in ETE Delay.

End to End Delay for Router 1: In the graph (Fig. 6d) the red line shows ETE Delay for Router 1 for Tree_Routing while the blue line indicates for Mesh_Routing. Once again as before, the ETE Delay in Mesh_Routing is lower due to ability of mesh routing process to find more efficient route (1 hop vs. 2 hops).

Global Mac Load per PAN: In the graph (Fig. 6c) the red line is the total load for the Tree_Routing scenario while the blue line is total load for Mesh_Routing. The load for Mesh_Routing is lower due to fewer hops for application traffic resulting in overall less traffic seen at the MAC layer. Also there is a small spike in load for Mesh_Routing near the beginning of the simulation. This is due to the routing messages being broadcast at that time.

\section{Roaming in PANs}

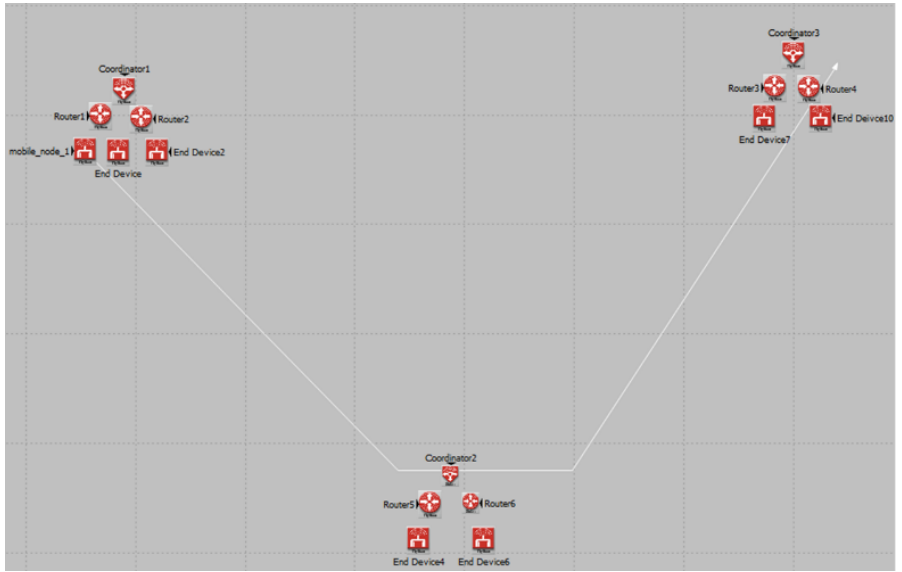

Fig. 7. This scenario studies the behavior of a mobile ZigBee node passing through the radius of multiple PANs 
Paper-Investigation and Performance Analysis of Some Implemented Features of the ZigBee Protocol and IEEE 802.15.4 Mac Specification

\section{Results:}

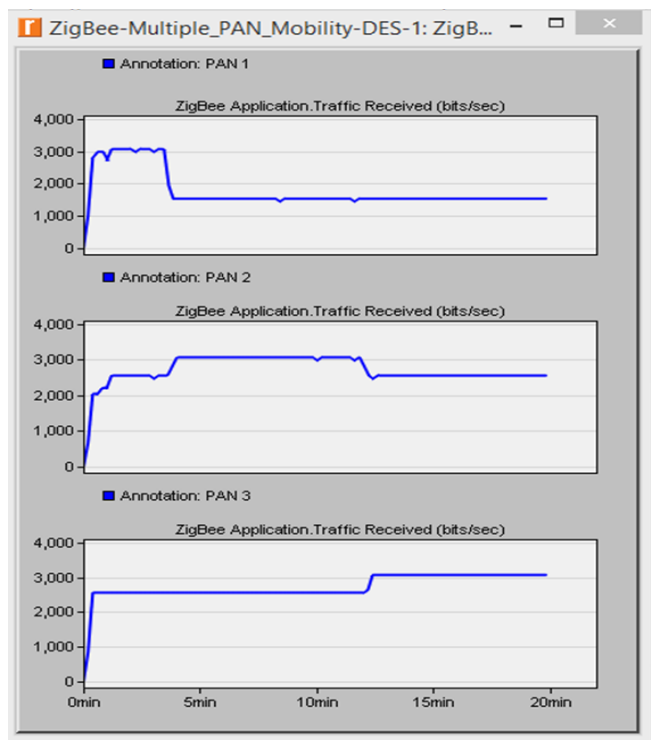

a) Showing Multiple PAN mobility

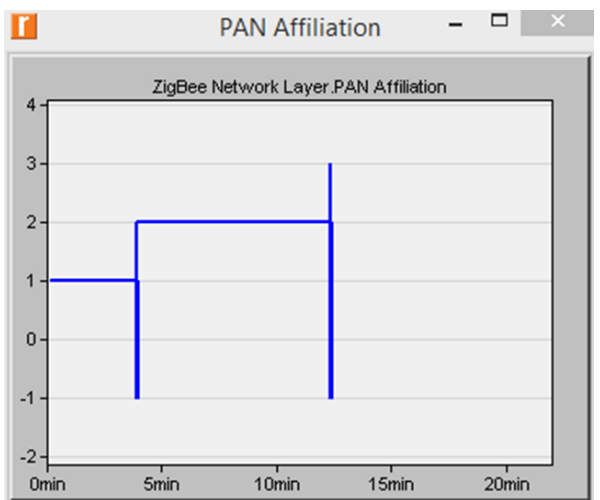

b) PAN affiliation

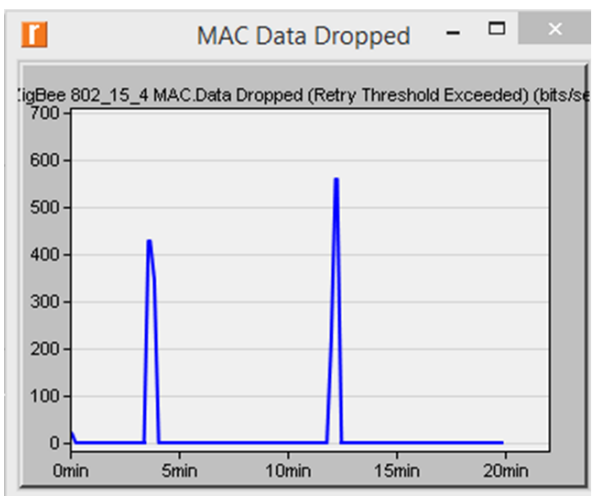

c) MAC data drop

Fig. 8.

PAN Affiliation for mobile_node_1: The graph (Fig. 8b) shows mobile_node_1 is connected to PAN 1 for the first 4 minutes of the simulation. Then the node briefly disconnects from the PAN (PAN ID -1), then promptly connects to PAN 2. After 12 minutes, the node disconnects from PAN 2 and promptly connects to PAN 3.

Data Dropped (Retry Threshold Exceeded) for mobile_node_1: The graph (Fig. 8c) shows that there were no data packets dropped for most of the simulation, with only brief spikes at around 4 and 12 minutes. Closer examination shows that each 
Paper-Investigation and Performance Analysis of Some Implemented Features of the ZigBee Protocol and IEEE 802.15.4 Mac Specification

spike occurs just before the node switches PANs, during the time when it is out of range of its parent but has not yet left the PAN.

Global Application Traffic Received for PAN 1, 2, and 3: The traffic received graphs (Fig. 8a) for each PAN are as expected. PAN 1's traffic is initially higher as it has an additional node (mobile_node_1). When mobile_node_1 switches to PAN 2, the traffic received for PAN 1 decreases while the traffic received for PAN 2 increases. A similar thing happens again for PANs 2 and 3 at around 12 minutes, when mobile_node_1 switches PANs again.

\section{Failure and Recovery procedures:}

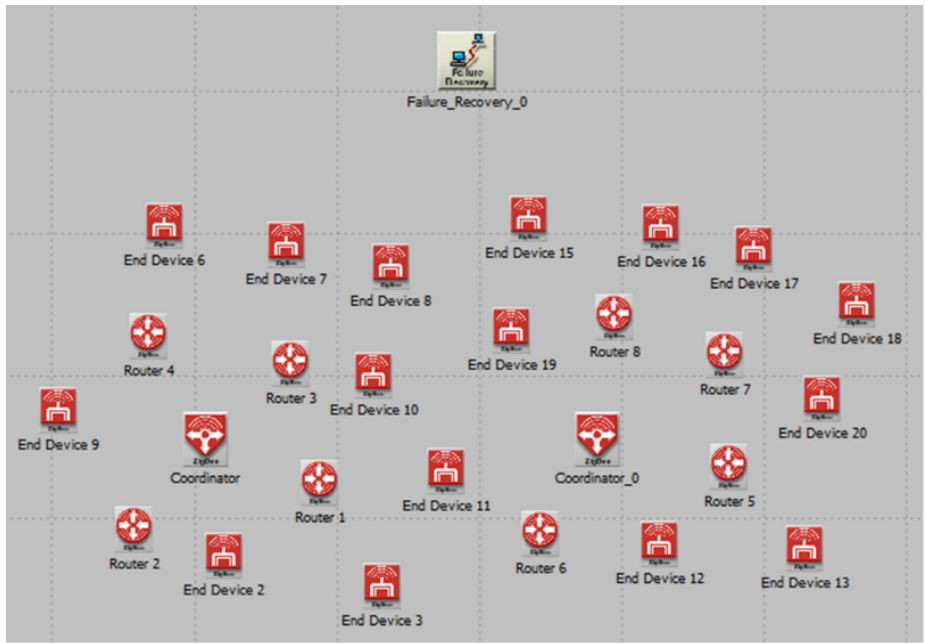

Fig. 9. This scenario studies the behavior of a ZigBee network when the coordinator fails. [25]

\section{Results:}

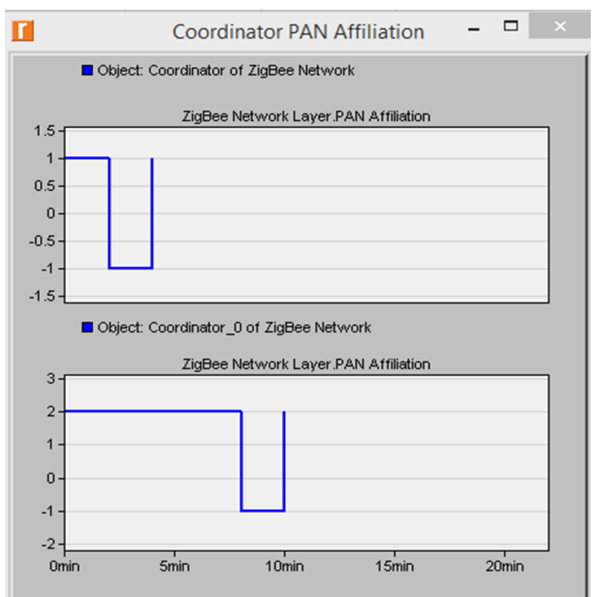

a) Coordination PAN Affiliation

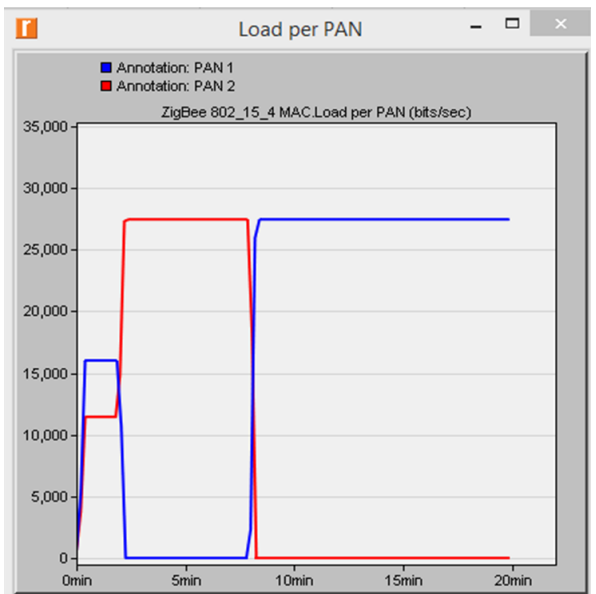

b) Load per PAN

Fig. 10. 
Paper-Investigation and Performance Analysis of Some Implemented Features of the ZigBee Protocol and IEEE 802.15.4 Mac Specification

Global MAC load per PAN: In the graph (Fig. 10b) the blue line is the first coordinator (PAN 1) while the red is the second coordinator (PAN 2). Initially, both PANs have almost equivalent loads (PAN 1's is greater due to a few more nodes joining that network). After 2 minutes, PAN 1's load drops to zero while PAN 2's load increases. Note that these numbers remain constant even after the first coordinator recovers at 4 minutes. After 8 minutes when the second coordinator fails, PAN 1's load increases while PAN 2's falls to zero.

PAN Affiliation for Coordinator and Coordinator_0: From these two graphs (Fig. 10a) it can be seen that during their failed periods the coordinators have a PAN ID of -1 , which is the code indicating that they are not currently connected to the network.

Global Output Report: In the "view results browser", the "DES Run (1) Tables" tab was opened then the ZigBee list item was expanded. From there the Number of Nodes column in the three tables it was seen that initially 15 nodes were joined to PAN 1 while 11 were connected to PAN 2 (roughly half, with an acceptable variation due to randomness). After the first failure, 25 nodes were connected to PAN 2 while only one (the coordinator itself) was connected to PAN 1. After the second failure, the connections were just reversed.

\section{Discussion and Conclusion}

\subsection{Discussions}

ZigBee is appropriate for basic applications within a home that send small packets of data, such as a passive sensor or a light switch. These devices are of low-rate WPAN (LR-WPAN) specifications, and use minute power. ZigBee uses $2.4 \mathrm{GHz}$ worldwide, $868 \mathrm{MHz}$ in Europe, and $900 \mathrm{MHz}$ in the US.

There are some limitations of using OPNET Modeler. ZigBee gateway is unavailable as an object in the OPNET object palette. Another drawback is that OPNET does not provide any parameter change for distances. The only way to check if the range is correct is via trial \& error method. Therefore, developing a model of ZigBee Gateway and implementing it with the simulation package will be a useful research in the future.

No element is currently available for interfacing ZigBee PAN to the internet. Therefore, the possibility of using coordinator to Ethernet connection can also be tried out to see if it solves the problem. Future work might also include setting up a test-bed to compare the theoretical work done in OPNET with a practical implementation of the whole project. In that way, the model can be further improved by comparing the actual results with the two set-ups.

\subsection{Suggestion for future Work}

There are lots of other features in ZigBee Network and Physical parameters. Those parameters can also be tuned for having a greater performance of the ZigBee Network. 
Paper-Investigation and Performance Analysis of Some Implemented Features of the ZigBee Protocol and IEEE 802.15.4 Mac Specification

Throughout this project, some of the limitations about the ZigBee model in OPNET was learnt. It has been widely popularized that internet connection to a ZigBee network is a way which can make monitoring, observation, tracking and managing ZigBee controlled environment smoother \& meaningful. Indeed, there are applications in agriculture, health, industry, building facilities and automation which can be only possible if there is an interface between ZigBee network \& IP-based internet. As per ZigBee alliance "The Gateway specification defines a Remote Procedure Call-based (RPC) API to ZigBee functionality and the management of the IP gateway itself". ZigBee was designed with expectations of low cost, long battery life, low duty cycles and smaller sizes. The protocol stack was also designed so that the processing power required to run the stack fitted just inside the hardware specifications, there were no heavy data communication protocols like IP / TCP /UDP included. Absence of such IP interfacing protocols made it really difficult to connect ZigBee environment with the Internet in the simulation model. When the Internet connection was implemented, it was noticed that OPNET doesn't support ZGD (ZigBee Gateway Device).

\subsection{Conclusions}

ZigBee contains lots of benefits such as it supports various network topologies, such as point to point, point to multiple and mesh networks. It has low duty cycle, leading to greater battery life, low latency and direct sequence spread spectrum and has almost up to 65000 nodes per network. It can access channels using Carrier Sense Multiple Access with Collision Avoidance (CSMA-CA) and contains 128-bit AES encryption for secured data connections.

Some of the current applications of ZigBee are in designing of intelligent warehouse Measure and Control System Based on Zigbee WSN, ZigBee Based MultiLevel Parking Vacancy Monitoring System [27], design of Greenhouse Monitoring \& Control System Based on ZigBee Wireless Sensor Network [28], Research of Wireless Sensor Networks based on ZigBee for Miner Position [29] Applications of Wireless Sensor Networks in Environmental Monitoring [9]. Also some of the basic applications supported are Home Automation and Control, Automatic Meter Reading, Residential \& commercial utility systems, Building Automation, Body area networks, Fitness monitoring: home, gym, ZigBee Smart Energy, Patient monitoring, Cable replacements, Automotive, In vehicle control: vehicular \& entertainment, Status monitoring, Telecom Services [8]. Even though the running cost is low it is still quite high to set up compared to other technologies as new equipment would be needed for it. ZigBee is still not widespread so there are limited end devices available thus even though it has the potential it is not being utilized properly. So if the use of ZigBee was increased in different networking devices, it can be used to easily control devices connected to it. Unfortunately, Zigbee has low bandwidth $(250 \mathrm{kbps})$ thus it can only be used for low data transfer applications and is prone to various network attack and penetration for which it is not recommended for office use [26]. So many different companies such as ZigBee Alliance is working on making it more secured and developing more software for it. 
Paper-Investigation and Performance Analysis of Some Implemented Features of the ZigBee Protocol and IEEE 802.15.4 Mac Specification

\section{References}

1. Farahani S. "ZigBee wireless networks and Transceivers: The complete guide for RF/wireless engineers". Amsterdam: Newnes (an imprint of Butterworth-Heinemann Ltd); October 6, 2008.

2. Alnuaimi M, Shuaib K, Jawhar I. "Performance evaluation of IEEE 802.15.4 physical layer using MatLab/Simulink". 2006 Innovations in Information Technology. November 2006: p1-5. https://doi.org/10.1109/INNOVATIONS.2006.301905

3. Chen C. "Zigbee Smart Energy Products. Colorado: Webcom Communication Corp".; August 31, 2015: p15-16. http://www.nxtbook.com/nxtbooks/webcom/zigbee_rg2015. Accessed October 10, 2016.

4. Archana R.Raut and Dr L. G. Malik "ZigBee: The Emerging Technology in Building Automation", International Journal of Computer Science and Engineering(IJCSE), Vol.3, No.4, April 2011, p1479-1484

5. Lee J-S, Su Y-W, Shen C-C. "A comparative study of wireless protocols: Bluetooth, UWB, ZigBee, and Wi-Fi. IECON 2007" - 33rd Annual Conference of the IEEE Industrial Electronics Society. November 2007: p46-51. https://doi.org/10.1109/IECON.2007. 4460126

6. Li Y, Zhang K, Zhang X. "Research on application of ZigBee technology in flammable and explosive environment". 2009 First International Conference on Information Science and Engineering. December 2009: p467-471 https://doi.org/10.1109/ICISE.2009.936

7. L. Jaiswal, J. Kaur and G. Singh, "Performance Analysis of Topological Variation in Personal Area Network using ZigBee Wireless Sensors", International Journal of Computer Science and Technology(IJCST), vol. 3, no. 4, December 2012, p706-711

8. Nisha Ashok Somani and Yash Patel, "ZigBee: A Low Power Wireless Technology for Industrial Appilication", International Journal of Control Theory and Computer Modelling (IJCTCM), Vol. 2, No.3, May 2012, p27-33 https://doi.org/10.5121/ijctcm.2012.2303

9. Ye D, Gong D, Wang W. "Application of wireless sensor networks in environmental monitoring”. 2009 2nd International Conference on Power Electronics and Intelligent Transportation System (PEITS). December 2009: p2563-2567. https://doi.org/10.1109/PEITS. 2009.5407035

10. Zhang X, Han G, Zhu C, Dou Y, Tao J. "Research of wireless sensor networks based on ZigBee for miner position". 2010 International Symposium on Computer, Communication, Control and Automation (3CA). May 2010: p1-7. https://doi.org/10.1109/3CA.2010. $\underline{5333883}$

11. Manpreet, Jyoteesh Malhotra, "Simulation Analysis of Tree and Mesh Topologies in ZigBee Network", International Journal of Grid Distribution Computing, vol.8, no.1, 2015, p81-82. https://doi.org/10.14257/ijgdc.2015.8.1.08

12. Nahla Abdulrahman Hussain, Fuad Ali Yaseen, "Performance Evaluation of ZigBee Routing Protocol under Various Condition using OPNET Moduler", International Journal of Computer Applications (0975- 8887), vol. 117, no. 18, May 2015, p25-31

13. Zhou M, Nie Z. "Analysis and design of ZigBee MAC layers protocol". 2010 International Conference on Future Information Technology and Management Engineering. 2010; Vol. 2: p211-215. https://doi.org/10.1109/FITME.2010.5654824

14. Kalaivani T, Allirani A, Priya P. "A survey on Zigbee based wireless sensor networks in agriculture". 3rd International Conference on Trendz in Information Sciences \& Computing (TISC2011). December 2011. https://doi.org/10.1109/TISC.2011.6169090 
Paper-Investigation and Performance Analysis of Some Implemented Features of the ZigBee Protocol and IEEE 802.15.4 Mac Specification

15. Md. Mamunur Rashid and Rethwan Faiz. "ZigBee: Simulation and Investigation of Star and Mesh Topology by using different Transmission Bands". The AIUB Journal of Science and Engineering (AJSE). Vol. 14 No. 1, August 2015, p115-121.

16. Ms. Dharmistha D.Vishwakarma, "IEEE 802.15.4 and ZigBee: A Conceptual Study", International Journal of Advanced Research in Computer and Communication Engineering, Vol 1, No 7. September 2012, p477-480.

17. Thoraya Obaid, Haleemah Rashed, Ali Abou-Elnour, Muhammad Rehan, Mussab Muhammad Saleh and Mohammed Tarique, "ZigBee Technology and its Application in Wireless Home Automation System: A Survey", International Journal of Computer Networks and Communication (IJCNC), Vol 6, No 4, July 2014, p115-131. https://doi.org/10.5121/ ijcnc.2014.6411

18. OPNET Technologies Co. OPNET technologies. http://www.opnet.com.tw/about.html. Accessed October 10, 2016.

19. Hammoodi IS, Stewart BG, Kocian A, McMeekin SG. "A comprehensive performance study of OPNET Modeler for ZigBee wireless sensor networks". 2009 Third International Conference on Next Generation Mobile Applications, Services and Technologies. September 2009. https://doi.org/10.1109/NGMAST.2009.12

20. "Part 15.4: Wireless Medium Access Control (MAC) and Physical Layer (PHY) Specifications for Low-Rate Wireless Personal Area Networks (WPANs)". 2nd ed. New York, NY: Institute of Electrical and Electronics Engineers; June 06, 2006.

21. Prativa P. Saraswala, "A Survey on Routing Protocols in ZigBee Network", International Journal of Engineering Science and Innovation Technology, Vol. 2, No 1 January 2013, p471-476.

22. Amritpal Kaur, Jaswinder Kaur and Gurjeevan Singh, "Modeling and Simulation of CSMA/CA Slotted and Unslotted Mode in Zigbee Routing Schemes", International Journal of Computer Applications, Vol. 103, No.7, October 2014, p40-43 https://doi.org/10.5120/18090-9142

23. Mohammed.I. Benakila, Laurent George, Smain Femmam, "A Beacon Cluster-Tree Construction Approach for ZigBee/IEEE802.15.4 Networks", UBICOMM 2010: The Fourth International Conference on Mobile Ubiquitous Computing, Systems, Services and Technologies. October 2010, p130-138.

24. Sinem Coleri Ergen. "IEEE 802.15.4 PHY" in ZigBee/IEEE 802.15.4 Summary. Vol. 1, No. 3, September 2004, p7-9.

25. Prof. Satish K Shah, Ms Sonal J. Rane, Ms Dharmishtha D. Vishwakarma. "Analytical Approach for Performance of Wireless Sensor Networks". International Journal of Electronics and Computer Science Engineering(IJECSE), Vol. 1, January 2013, p1877-1884

26. Akshay Kanwar, Aditi Khazanchi. "ZigBee: The New Bluetooth Technology". International Journal Of Engineering And Computer Science. Vol. 1, No. 2, November 2012, p6774.

27. Vishnubhotla R, Rao PS, Ladha A, et al. "ZigBee based multi-level parking vacancy monitoring system." 2010 IEEE International

28. Conference on Electro/Information Technology. May 2010: p2563-2566. https://doi.org/10.1109/EIT.2010.5612133

29. Zhou Y, Yang X, Guo X, Zhou M, Wang L. A design of greenhouse monitoring \& control system based on ZigBee wireless sensor network. 2007 International Conference on Wireless Communications, Networking and Mobile Computing. September 2007. https://doi.org/10.1109/WICOM.2007.638

30. Zhang X, Han G, Zhu C, Dou Y, Tao J. "Research of wireless sensor networks based on ZigBee for miner position." 2010 International Symposium on Computer, Communication, 
Paper-Investigation and Performance Analysis of Some Implemented Features of the ZigBee Protocol and IEEE 802.15.4 Mac Specification

Control and Automation (3CA). May 2010: p1-5. https://doi.org/10.1109/3CA.2010. 5533883

31. Tariq, S. "MAC algorithms in wireless networks: applications, issues and comparisons". Diss. Master thesis, Department of Computing Science, Umea University, Sweden, 2005.

32. Vutukuri A, Bhattacharya S, Raj T, Sridhar, Geetha V. "Enhanced back-off technique for IEEE 802.15.4 WSN standard." In: Meghanathan N, Nagamalai D, Rajasekaran S, eds. Networks and Communications (NetCom2013). Switzerland: Springer International Publishing; January 2014: p21-29. https://doi.org/10.1007/978-3-319-03692-2 2

33. Rao VP, Marandin D. "Adaptive Backoff Exponent algorithm for Zigbee (IEEE 802.15.4)." In: Koucheryavy Y, Harju J, Iversen VB, eds. Next Generation Teletraffic and Wired/Wireless Advanced Networking. Germany: Springer Berlin Heidelberg; 2006: p501-516. https://doi.org/10.1007/11759355 46

34. Xiao Y, Shen X, Jiang H. "Optimal ACK mechanisms of the IEEE 802.15.3 MAC for ultra-wideband systems." IEEE Journal on Selected Areas in Communications. 2006;24(4): p836-842. https://doi.org/10.1109/JSAC.2005.863862

\section{Authors}

Wayesh Qarony is a senior lecturer (on leave) in Electrical and Electronic Engineering Department, American International University (AIUB), Kemal Ataturk Avenue Banani, Dhaka 1213, Bangladesh. He is now with the Department of Applied Physics in The Hong Kong Polytechnic University, Hong Kong being a PhD fellow. (wayesh@aiub.edu).

Sayera Aznabi graduated from AIUB in the department of Electrical and Electronic Engineering (aznabis@yahoo.com).

N. Tahsina Islam graduated from AIUB in the department of Electrical and Electronic Engineering (nabila.tahsina.islam@gmail.com).

S. K. Nath is a lecturer of AIUB in the department of Electrical and Electronic Engineering (sknath@aiub.edu).

Annan Faridi is an undergraduate student from AIUB in the department of Electrical and Electronic Engineering (annan.faridi@gmail.com).

Submitted 24 June 2016. Published as resubmitted by the authors 12 October 2016. 